\title{
SYNTHESIS, CHARACTERIZATION AND ANTI- INFLAMMATORY ACTIVITY OF CERTAIN NOVEL 2, 4- DISUBSTITUTED 1,3-THIAZOLE DERIVATIVES
}

\author{
P. Gobala Krishnan ${ }^{1, *}$, K. Gnana Prakash ${ }^{2}$ and K. B. Chandra Sekhar ${ }^{3}$ \\ ${ }^{1}$ Research Scholar, JNTUA, Ananthapuramu, Andhra Pradesh- 515 002, India. \\ ${ }^{2}$ Department of Pharmaceutical chemistry, Saastra college of Pharmaceutical Education and \\ Research, Nellore - 524 311, Andhra Pradesh, India. \\ ${ }^{3}$ Department of Organic Chemistry, JNTUA Oil Technology and Pharmaceutical Research \\ Institute, Ananthapuramu -515 001, Andhra Pradesh India. \\ *E-mail: gopalintas2004@gmail.com
}

\begin{abstract}
A series of 2,4-disubstituted thiazole derivatives (6a-k) were synthesized by a condensation reaction between 2amino, 4-substituted phenyl thiazole and 2-(2,3-dimethylanilino)benzoic acid. The structure of synthesized thiazole derivatives 6(a-k) were characterized by using IR, NMR, Mass Spectroscopy and evaluated for their in vitro antiinflammatory activity using albumin denaturation method and compared with standard drug diclofenac sodium. The compounds 2-(2,3-dimethyl phenylamino)-N-(4-(4-methyl phenyl) thiazol-2-yl)benzamide (6a) and 2-(2,3-dimethyl phenylamino)-N-(4-(4-nitrophenyl) thiazol-2-yl)benzamide (6c) showed better anti-inflammatory activity when compared with a standard drug.
\end{abstract}

Keywords: 2-amino 4-phenyl thiazole, 2-(2,3-dimethylanilino)benzoic acid, albumin denaturation method, antiinflammatory activity.

(C) RASĀYAN. All rights reserved

\section{INTRODUCTION}

Thiazoles are aromatic, five-membered heterocyclic ring system containing sulphur and nitrogen placed in the heterocyclic ring at 1,3 position and is found in various natural and synthetic drugs. They are isomeric with 1, 2-azoles. The numbering of the thiazole ring system is shown below.

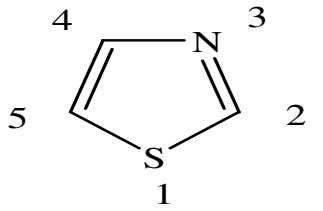

Numbering system of thiazole ring system

Fig.-1

Thiazoles are important leading molecules for designing potential bioactive drugs. Thiazole ring is an integral part of penicillin antibiotics which have a revolutionized therapy in bacterial diseases. It also presents in the structure of many natural products and biologically active compounds, like thiamine (vitamin B1) and many metabolic products of fungi and primitive marine animal etc.

Thiazoles were found in the drug development for the treatment of various diseases like allergies, hypertension, inflammation, schizophrenia, bacterial, HIV infections, hypnotics, and also in some antibiotic drugs like penicillin, micrococcin due to the presence of $(\mathrm{S}-\mathrm{C}=\mathrm{N})$ unit. ${ }^{1-3}$ Literature survey reveals that thiazoles were reported in various therapeutic areas such as antimicrobial, antitubercular, anticonvulsant, anticancer, anti-HIV, analgesic, cardiotonic and anti-inflammatory activities. ${ }^{4-10}$

Rasayan J. Chem., 12(1), 366-371(2019)

http://dx.doi.org/10.31788/RJC.2019.1215069

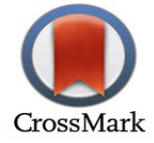


Nonsteroidal anti-inflammatory drugs (NSAIDS) such as indomethacin and diclofenac are broadly used therapeutic drugs, primarily for pain, inflammation and arthritis. The beneficial anti-inflammatory and analgesic effects of NSAIDs is due to their inhibition of cycloxigenase (COX) thereby inhibiting prostaglandin synthesis. Substituted thiazole compounds especially 2-amino thiazoles are the most important building blocks in recent drug discovery and are found in various biological activities. In continuation of these, it was decided to synthesize certain novel 2,4-disubstituted thiazole derivatives and evaluated for anti-inflammatory activity.

\section{EXPERIMENTAL}

\section{Materials and Methods}

Chemicals were purchased from Siga-Aldrich and Sd fine chemicals Ltd as Synthesis Grade and used without further purification. Melting points of the synthesized compounds were determined using the Thermionic Melting point apparatus and were found uncorrected. All the synthesized compounds were characterized by IR, NMR and Mass spectroscopy. All the compounds were screened for their in-vitro anti-inflammatory activity by using the albumin denaturation method.

\section{Synthesis of 2,4- Disubstituted Thiazoles (6a-k)}

The scheme of synthesis of 2,4-disubstituted thiazole derivatives is shown in Figure: 2 and list of substituent and their physical properties were listed in Table-1.

\section{Synthesis of 2-Amino 4-Substituted Phenyl Thiazole Derivatives (3a-k)}

A round bottom flask was charged with $2.0 \mathrm{~g}$ of substituted acetophenone ( 0.016 moles $), 2.5 \mathrm{~g}$ of thiourea (0.033 mole), $2.01 \mathrm{~g}$ of Iodine $(0.016$ mole $)$ and $50 \mathrm{ml}$ of absolute ethanol. The reaction mixture was refluxed for overnight. The mixture was diluted with $50 \mathrm{ml}$ of water and heated further to dissolve the solid and filtered. The filtrate was cooled in ice mixture, basified with aqueous ammonia and the resulting precipitate was filtered, washed with diethyl ether and recrystallized from ethanol. ${ }^{11}$

\section{Synthesis of 2-(2,3-dimethylanilino)-N-(4-substitutedphenyl-1,3-thiazol-2-yl)benzamides (6a-k)}

The compound 2-(2,3-dimethylanilino)benzoic acid $(0.2 \mathrm{~g}, 0.00082 \mathrm{~mol})$ and 2-amino-4-phenyl thiazole $0.174 \mathrm{~g}(0.099 \mathrm{~mol})$ were dissolved in dichloromethane $(5 \mathrm{~mL})$ into this 4-Dimethylaminopyridine (DMAP) were added with continuous stirring at $0^{\circ} \mathrm{C}$ for $30 \mathrm{mins}$. To this solution, DCC (N,N'dicyclohexylcarbodiimide) was added and stirred for $15 \mathrm{hrs}$ under a nitrogen atmosphere. The completion of the reaction was monitored by TLC and the reaction mixture was extracted with dichloromethane. The organic layer was washed with sodium bicarbonate solution, water, brine solution, which was separated and dried over anhydrous sodium sulphate. The evaporation of solvent yielded target compounds. ${ }^{12}$

\section{Spectral Characterizations}

\section{2-(2,3-dimethylphenylamino)-N-(4-(4-methylphenyl) thiazol-2-yl) benzamide (6a)}

Yellow solid (66\%); mp 184-186 ${ }^{\circ} \mathrm{C}$; FT-IR $\left(\mathrm{KBr}, \mathrm{cm}^{-1}\right)$ : $3550(\mathrm{~N}-\mathrm{H}) ; 2942(\mathrm{Ar}-\mathrm{H}), 1666(\mathrm{C}=\mathrm{O}), 1570$ $(\mathrm{C}=\mathrm{N}), 1605$ (C=C), 729 (C-S-C). ${ }^{1} \mathrm{H}-\mathrm{NMR}$ (400 MHz, DMSO-d6) ppm: 2.09 (s, 3H, CH $)$; 2.28 (s, 3H, $\left.\mathrm{CH}_{3}\right) ; 2.35\left(\mathrm{~s}, 3 \mathrm{H}, \mathrm{CH}_{3}\right) ; 6.67-6.70(\mathrm{t}, 2 \mathrm{H}, \mathrm{Ar}-\mathrm{H}) ; 7.01-7.05(\mathrm{~m}, 2 \mathrm{H}, \mathrm{Ar}-\mathrm{H}) ; 7.09$ - 7.13 (t, 2H, Ar-H); $7.28-7.37$ (m, 4H, Ar-H); 7.51 - 7.56 (t, 1H, Ar-H); 7.86 - 7.89 (d, 1H, Ar-H); 9.44 (s, 1H, NH). MS: $\mathrm{m} / \mathrm{z} 414.2(\mathrm{M}+1)$.

\section{2-(2,3-dimethylphenylamino)-N-(4-(4-methoxyphenyl) thiazol-2-yl)benzamide (6b)}

Pale yellow solid (84\%); mp 186-188 ${ }^{\circ} \mathrm{C}$; FT-IR (KBr, cm-1): $3318(\mathrm{~N}-\mathrm{H})$; 2991 (Ar-H), 2941 (C-H), $1650(\mathrm{C}=\mathrm{O}), 1575(\mathrm{C}=\mathrm{N}), 1450(\mathrm{C}=\mathrm{C}), 1388(\mathrm{C}-\mathrm{N}) 729(\mathrm{C}-\mathrm{S}-\mathrm{C}) .{ }^{1} \mathrm{H}-\mathrm{NMR}$ (400 MHz, DMSO-d6) ppm: $2.10\left(\mathrm{~s}, 3 \mathrm{H}, \mathrm{CH}_{3}\right) ; 2.28\left(\mathrm{~s}, 3 \mathrm{H}, \mathrm{CH}_{3}\right) ; 3.82\left(\mathrm{~s}, 3 \mathrm{H}, \mathrm{OCH}_{3}\right) ; 6.67-6.71(\mathrm{t}, 2 \mathrm{H}, \mathrm{Ar}-\mathrm{H}) ; 6.67-6.71$ (t, $2 \mathrm{H}, \mathrm{Ar}-$ $\mathrm{H})$; 7.02-7.04 (m, 2H, Ar-H); $7.09-7.13(\mathrm{t}, 2 \mathrm{H}, \mathrm{Ar}-\mathrm{H}) ; 7.29-7.32(\mathrm{~m}, 4 \mathrm{H}, \mathrm{Ar}-\mathrm{H}) ; 7.40$ - $7.44(\mathrm{t}, 1 \mathrm{H}$, Ar-H); $7.88-7.89$ (d, 1H, Ar-H); 8.71 (s, 1H, NH); 9.46 (s, 1H, NH). MS: m/z 430.2 (M+1).

\section{2-(2,3-dimethylphenylamino)-N-(4-(4-nitro phenyl) thiazol-2-yl)benzamide (6c)}

Yellow solid (82\%); mp 207-209 ${ }^{\circ} \mathrm{C}$; FT-IR (KBr, cm $\left.{ }^{-1}\right): 3341$ (N-H); 2989 (Ar-H), 1644 (C=O), 1574 $(\mathrm{C}=\mathrm{N}), 1451(\mathrm{C}=\mathrm{C}), 1390(\mathrm{C}-\mathrm{N}) 741$ (C-S-C). ${ }^{1} \mathrm{H}-\mathrm{NMR}$ (400 MHz, DMSO-d6) ppm: 2.09 (s, 3H, $\mathrm{CH}_{3}$ ); 2.29 (s, 3H, $\left.\mathrm{CH}_{3}\right) ; 6.67-6.71(\mathrm{t}, 2 \mathrm{H}, \mathrm{Ar}-\mathrm{H}) ; 7.02-7.05(\mathrm{~m}, 2 \mathrm{H}, \mathrm{Ar}-\mathrm{H}) ; 7.11$ - 7.12 (t, 2H, Ar-H); $7.28-$ 
RASĀYAN J. Chem.

Vol. 12 | No. 1 |366 - 371| January - March | 2019

7.33 (m, 4H, Ar-H); 7.43 (t, 1H, Ar-H); 7.86 - 7.89 (d, 1H, Ar-H); 8.23 (s, 1H, NH); 9.44 (s, 1H, NH). MS: $\mathrm{m} / \mathrm{z} 445.2(\mathrm{M}+1)$.

2-(2,3-dimethylphenylamino)-N-(4-(3,4,5-trimethoxyphenyl) thiazol-2-yl)benzamide (6d)

Yellow solid (68\%); mp 175-177 ${ }^{\circ} \mathrm{C}$; FT-IR $\left(\mathrm{KBr}, \mathrm{cm}^{-1}\right)$ : $3318(\mathrm{~N}-\mathrm{H}) ; 2923(\mathrm{Ar}-\mathrm{H}), 2941(\mathrm{C}-\mathrm{H}), 1672$ $(\mathrm{C}=\mathrm{O}), 1573(\mathrm{C}=\mathrm{N}), 1449(\mathrm{C}=\mathrm{C}), 1382(\mathrm{C}-\mathrm{N}) 735(\mathrm{C}-\mathrm{S}-\mathrm{C}) .{ }^{1} \mathrm{H}-\mathrm{NMR}$ (400 MHz, DMSO-d6) ppm: 2.09 $\left(\mathrm{s}, 3 \mathrm{H}, \mathrm{CH}_{3}\right) ; 2.28\left(\mathrm{~s}, 3 \mathrm{H}, \mathrm{CH}_{3}\right) ; 3.68\left(\mathrm{~s}, 3 \mathrm{H}, \mathrm{OCH}_{3}\right) ; 6.67-6.70(\mathrm{t}, 2 \mathrm{H}, \mathrm{Ar}-\mathrm{H}) ; 7.02-7.04(\mathrm{~m}, 2 \mathrm{H}, \mathrm{Ar}-\mathrm{H})$; 7.09 - 7.17 (t, 2H, Ar-H); 7.28 - 7.32 (m, 4H, Ar-H); 7.86 - 7.89 (d, 1H, Ar-H); 9.44 (s, 1H, NH). MS: $\mathrm{m} / \mathrm{z} 490.2(\mathrm{M}+1)$.

2-(2,3-dimethylphenylamino)-N-(4-(4-fluorophenyl) thiazol-2-yl)benzamide (6e)

Yellow solid (66\%); mp 179-181 ${ }^{\circ} \mathrm{C}$; FT-IR $\left(\mathrm{KBr}, \mathrm{cm}^{-1}\right)$ : $3359(\mathrm{~N}-\mathrm{H}) ; 2919(\mathrm{Ar}-\mathrm{H}), 1673(\mathrm{C}=\mathrm{O}), 1571$ $(\mathrm{C}=\mathrm{N}), 1448(\mathrm{C}=\mathrm{C}), 1381(\mathrm{C}-\mathrm{N}) 730(\mathrm{C}-\mathrm{S}-\mathrm{C}) .{ }^{1} \mathrm{H}-\mathrm{NMR}$ (400 MHz, DMSO-d6) ppm: 2.09 (s, 3H, $\left.\mathrm{CH}_{3}\right)$; $2.28\left(\mathrm{~s}, 3 \mathrm{H}, \mathrm{CH}_{3}\right) ; 6.67-6.71(\mathrm{t}, 2 \mathrm{H}, \mathrm{Ar}-\mathrm{H}) ; 7.02-7.04(\mathrm{~m}, 2 \mathrm{H}, \mathrm{Ar}-\mathrm{H}) ; 7.08$ - $7.12(\mathrm{t}, 2 \mathrm{H}, \mathrm{Ar}-\mathrm{H}) ; 7.26-$ 7.33 (m, 4H, Ar-H); $7.86-7.89$ (d, 1H, Ar-H); 9.44 (s, 1H, NH). MS: m/z 430.2 (M+1).

2-(2,3-dimethylphenylamino)-N-(4-(3-methoxyphenyl) thiazol-2-yl)benzamide (6f)

Yellow solid (70\%); mp 194-196 ${ }^{\circ} \mathrm{C}$; FT-IR (KBr, cm $\left.{ }^{-1}\right)$ : $3366(\mathrm{~N}-\mathrm{H}) ; 2928(\mathrm{Ar}-\mathrm{H}), 1675$ (C=O), 1573 $(\mathrm{C}=\mathrm{N}), 1450(\mathrm{C}=\mathrm{C}), 1377$ (C-N) 733 (C-S-C). ${ }^{1} \mathrm{H}-\mathrm{NMR}$ (400 MHz, DMSO-d6) ppm: 2.09 (s, 3H, CH$)_{3}$; $2.29\left(\mathrm{~s}, 3 \mathrm{H}, \mathrm{CH}_{3}\right) ; 3.79\left(\mathrm{~s}, 3 \mathrm{H}, \mathrm{OCH}_{3}\right) ; 6.67-6.71(\mathrm{t}, 2 \mathrm{H}, \mathrm{Ar}-\mathrm{H}) ; 6.99(\mathrm{t}, 2 \mathrm{H}, \mathrm{Ar}-\mathrm{H}) ; 7.00-7.04(\mathrm{~m}, 2 \mathrm{H}, \mathrm{Ar}-$ $\mathrm{H})$; 7.11-7.12 (t, 2H, Ar-H); 7.28 - $7.33(\mathrm{~m}, 4 \mathrm{H}, \mathrm{Ar}-\mathrm{H}) ; 7.66-7.68(\mathrm{t}, 1 \mathrm{H}, \mathrm{Ar}-\mathrm{H}) ; 7.86-7.89$ (d, 1H, ArH); 9.44 (s, 1H, NH). MS: m/z $430.2(\mathrm{M}+1)$.

2-(2,3-dimethylphenylamino)-N-(4-(4-hydroxyphenyl) thiazol-2-yl)benzamide (6g)

Yellow solid (60\%); mp 208-210 ${ }^{\circ} \mathrm{C}$; FT-IR $\left(\mathrm{KBr}, \mathrm{cm}^{-1}\right)$ : $3318(\mathrm{~N}-\mathrm{H}) ; 2996(\mathrm{Ar}-\mathrm{H}), 2940(\mathrm{C}-\mathrm{H}), 1655$ $(\mathrm{C}=\mathrm{O}), 1573(\mathrm{C}=\mathrm{N}), 1448(\mathrm{C}=\mathrm{C}), 1383(\mathrm{C}-\mathrm{N}) 735$ (C-S-C). ${ }^{1} \mathrm{H}-\mathrm{NMR}$ (400 MHz, DMSO-d6) ppm: 2.09 $\left(\mathrm{s}, 3 \mathrm{H}, \mathrm{CH}_{3}\right) ; 2.28\left(\mathrm{~s}, 3 \mathrm{H}, \mathrm{CH}_{3}\right) ; 6.70(\mathrm{~s}, 1 \mathrm{H}, \mathrm{OH}) ; 6.84-6.86(\mathrm{t}, 2 \mathrm{H}, \mathrm{Ar}-\mathrm{H}) ; 6.95(\mathrm{t}, 2 \mathrm{H}, \mathrm{Ar}-\mathrm{H}) ; 7.01-7.03$ (m, 2H, Ar-H); 7.11 - 7.12 (t, 2H, Ar-H); 7.28 - 7.32 (m, 4H, Ar-H); 7.52-7.54 (t, 1H, Ar-H); 7.86-7.89 (d, 1H, Ar-H); 8.62 (s, 1H, NH); 9.44 (s, 1H, NH); 9.87 (s, 1H, NH). MS: m/z 416.2 (M+1).

2-(2,3-dimethylphenylamino)-N-(4-(4-bromophenyl)thiazol-2-yl)benzamide (6h)

Yellow solid (64\%); mp 198-200 ${ }^{\circ} \mathrm{C}$; FT-IR $\left(\mathrm{KBr}, \mathrm{cm}^{-1}\right)$ : $334(\mathrm{~N}-\mathrm{H}) ; 2919(\mathrm{Ar}-\mathrm{H}), 1648(\mathrm{C}=\mathrm{O}), 1572$ $(\mathrm{C}=\mathrm{N}), 1449(\mathrm{C}=\mathrm{C}), 1388$ (C-N) 731 (C-S-C). ${ }^{1} \mathrm{H}-\mathrm{NMR}$ (400 MHz, DMSO-d6) ppm: 2.09 (s, 3H, $\mathrm{CH}_{3}$ ); $2.28\left(\mathrm{~s}, 3 \mathrm{H}, \mathrm{CH}_{3}\right) ; 6.67-6.71\left(\mathrm{~s}, 3 \mathrm{H}, \mathrm{CH}_{3}\right) ; 7.02-7.05(\mathrm{t}, 2 \mathrm{H}, \mathrm{Ar}-\mathrm{H}) ; 7.11-7.12(\mathrm{t}, 2 \mathrm{H}, \mathrm{Ar}-\mathrm{H}) ; 7.28-7.33(\mathrm{~m}$, 2H, Ar-H); 7.57,7.59 (t, 2H, Ar-H); 7.72,7.74 (m, 4H, Ar-H); 7.86 - 7.89 (t, 1H, Ar-H); 9.44 (d, 1H, Ar$\mathrm{H}) ; 9.46$ (s, 1H, NH). MS: m/z $479.2(\mathrm{M}+1)$.

2-(2,3-dimethylphenylamino)-N-(4-(4-chlorophenyl)thiazol-2-yl)benzamide (6i)

Yellow solid (68\%); mp 197-199 ${ }^{\circ} \mathrm{C}$; FT-IR $\left(\mathrm{KBr}, \mathrm{cm}^{-1}\right)$ : $3365(\mathrm{~N}-\mathrm{H}) ; 2991$ (Ar-H), 2941 (C-H), 1673 $(\mathrm{C}=\mathrm{O}), 1573(\mathrm{C}=\mathrm{N}), 1450(\mathrm{C}=\mathrm{C}), 1380(\mathrm{C}-\mathrm{N}) 735(\mathrm{C}-\mathrm{S}-\mathrm{C}) .{ }^{1} \mathrm{H}-\mathrm{NMR}$ (400 MHz, DMSO-d6) ppm: 2.09 $\left(\mathrm{s}, 3 \mathrm{H}, \mathrm{CH}_{3}\right) ; 2.28\left(\mathrm{~s}, 3 \mathrm{H}, \mathrm{CH}_{3}\right) ; 6.67-6.71(\mathrm{t}, 2 \mathrm{H}, \mathrm{Ar}-\mathrm{H}) ; 7.01-7.04(\mathrm{~m}, 2 \mathrm{H}, \mathrm{Ar}-\mathrm{H})$; 7.11-7.12 (t, 2H, Ar$\mathrm{H}) ; 7.20$ (m, 4H, Ar-H); 7.28 - 7.32 (t, 1H, Ar-H); 7.50-7.52 (d, 1H, Ar-H); 7.86-7.89 (s, 1H, NH); 9.44 (s, 1H, NH). MS: m/z $434.2(\mathrm{M}+1)$.

2-(2,3-dimethylphenylamino)-N-(4-(4-hydroxy, 3-methoxy phenyl) thiazol-2-yl)benzamide (6j) Yellow solid (66\%); mp 218-220 ${ }^{\circ} \mathrm{C}$; FT-IR (KBr, cm $\left.{ }^{-1}\right): 3318(\mathrm{~N}-\mathrm{H}) ; 2924(\mathrm{Ar}-\mathrm{H}), 1650$ (C=O), 1572 $(\mathrm{C}=\mathrm{N}), 1449(\mathrm{C}=\mathrm{C}), 1382(\mathrm{C}-\mathrm{N}) 734$ (C-S-C). ${ }^{1} \mathrm{H}-\mathrm{NMR}$ (400 MHz, DMSO-d6) ppm: 2.09 (s, 3H, $\left.\mathrm{CH}_{3}\right)$; $2.28\left(\mathrm{~s}, 3 \mathrm{H}, \mathrm{CH}_{3}\right) ; 3.83\left(\mathrm{~s}, 3 \mathrm{H}, \mathrm{OCH}_{3}\right) ; 6.67-6.70(\mathrm{t}, 2 \mathrm{H}, \mathrm{Ar}-\mathrm{H}) ; 6.84-6.86(\mathrm{~m}, 2 \mathrm{H}, \mathrm{Ar}-\mathrm{H}) ; 7.00$ - $7.04(\mathrm{t}$, 2H, Ar-H); $7.09-7.14$ (m, 4H, Ar-H); 7.25 - 7.32 (t, 1H, Ar-H); $7.88-7.89$ (d, 1H, Ar-H); 8.62 (s, 1H, $\mathrm{NH}) ; 9.44$ (s, 1H, NH). MS: m/z 430.2 (M+1).

2-(2,3-dimethylphenylamino)-N-(4-(3,4-dimethoxyphenyl) thiazol-2-yl)benzamide (6k)

Pale yellow solid (68\%); mp 173-175 ${ }^{\circ} \mathrm{C}$; FT-IR $\left(\mathrm{KBr}, \mathrm{cm}^{-1}\right)$ : $3364(\mathrm{~N}-\mathrm{H}) ; 2925(\mathrm{Ar}-\mathrm{H}), 1674(\mathrm{C}=\mathrm{O})$, $1573(\mathrm{C}=\mathrm{N}), 1449(\mathrm{C}=\mathrm{C}), 1380(\mathrm{C}-\mathrm{N}) 733$ (C-S-C). ${ }^{1} \mathrm{H}-\mathrm{NMR}$ (400 MHz, DMSO-d6) ppm: 2.09 (s, 3H, 
$\left.\mathrm{CH}_{3}\right) ; 2.28\left(\mathrm{~s}, 3 \mathrm{H}, \mathrm{CH}_{3}\right) ; 3.79-3.81\left(\mathrm{~s}, 3 \mathrm{H}, \mathrm{OCH}_{3}\right) ; 6.67-6.71(\mathrm{t}, 2 \mathrm{H}, \mathrm{Ar}-\mathrm{H}) ; 7.02-7.04(\mathrm{~m}, 2 \mathrm{H}, \mathrm{Ar}-\mathrm{H})$; 7.11-7.12 (t, 2H, Ar-H); 7.27-7.33 (m, 4H, Ar-H); 7.86-7.89 (t, 1H, Ar-H); 9.44, 1H, NH); MS: m/z $460.2(\mathrm{M}+1)$.

\section{Chemistry}

\section{RESULTS AND DISCUSSION}

The present study was conducted to design, synthesize and evaluate anti-inflammatory activity of new 2,4-disubstituted thiazole derivatives as potential NSAIDs. A series of 2,4-disubstituted thiazole derivatives $(6 \mathrm{a}-\mathrm{k})$ were synthesized by a condensation reaction between 2-amino, 4-substituted phenyl thiazole (3a-k) and 2-(2,3-dimethylanilino)benzoic acid by using dimethylamino pyridine (DMAP) reagent. 2-amino, 4-substituted phenyl thiazole (3a-k) was synthesized by reaction of substituted acetophenone derivatives and thiourea in absolute ethanol, refluxed for overnight. All the synthesized derivatives were characterized by IR, ${ }^{1} \mathrm{H}$ NMR and Mass Spectroscopy and physicochemical properties of synthesized compounds were mentioned in Table-1.

\section{Step: 1 Synthesis of 2-Amino 4-Substituted Phenyl Thiazole Derivatives}
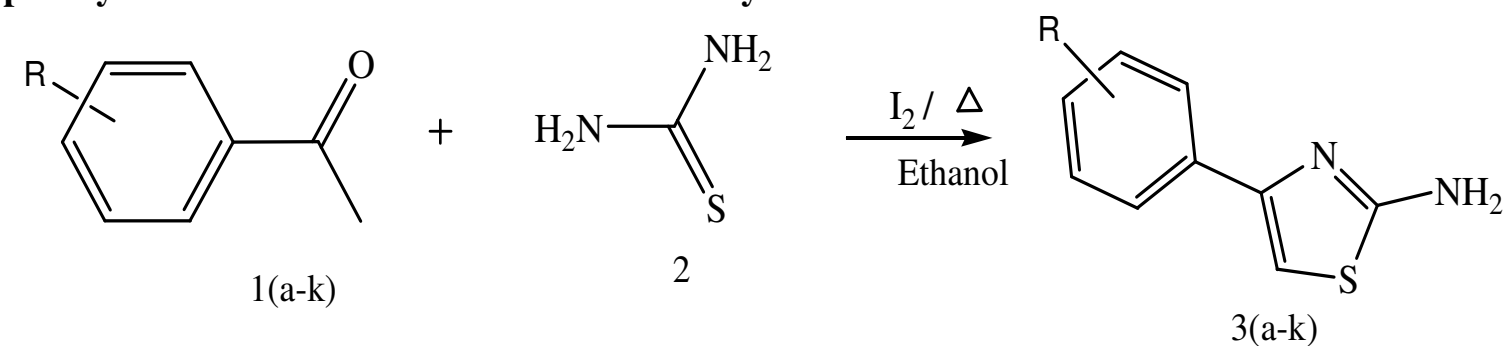

Step: 2 Synthesis of 2-(2,3-dimethylanilino)-N-(4-substituted phenyl-1,3-thiazol-2-yl) Benzamides<smiles>[R]c1ccc(-c2csc(NC(=O)c3ccccc3Nc3cccc(C)c3C)n2)cc1</smiles>

\section{Pharmacology}

Scheme-1

\section{Anti-inflammatory Activity}

The anti-inflammatory activity of all newly synthesized thiazole derivatives was carried out by the Albumin denaturation method. ${ }^{13}$ The standard drug and test compounds were dissolved by Dimethyl sulfoxide (DMSO). The standard and test sample containing different concentrations (50, 100, 200, 400, $800,1600 \mu \mathrm{g}$ ) were mixed with $1 \mathrm{ml}$ of $1 \mathrm{mM}$ albumin solution in phosphate buffer and incubated at $37^{\circ} \mathrm{C}$ for $15 \mathrm{~min}$. Add $3 \mathrm{ml}$ of phosphate buffer $(0.2 \mathrm{M}, \mathrm{PH} 7.4)$ and denaturation was induced by keeping the reaction mixture at $70^{\circ} \mathrm{C}$ in a water bath for $15 \mathrm{~min}$. After cooling, the turbidity was measured at $660 \mathrm{~nm}$. Percentage inhibition of protein denaturation was calculated from control, where no drug was added by using diclofenac sodium as a standard drug. The in vitro anti-inflammatory activity was calculated as $\%$ inhibition of protein denaturation and results are illustrated in Table-2 and Fig.-3. The percentage inhibition of protein denaturation was calculated by using the following formula:

$$
\% \text { inhibition of denaturation }=\frac{(\text { Absorbance of test }- \text { Absorbance of control })}{\text { Absorbance of test }} \times 100
$$

All the synthesized compounds were showed significant anti-inflammatory activities, among these the compounds 2-(2,3-dimethyl phenylamino)-N-(4-(4-methyl phenyl) thiazol-2-yl)benzamide (6a) and 2(2,3-dimethyl phenylamino)-N-(4-(4-nitrophenyl) thiazol-2-yl)benzamide (6c) showed better anti- 
RASĀYAN J. Chem.

Vol. 12 | No. 1 |366 - 371| January - March | 2019

inflammatory activities at $800 \mu \mathrm{g} / \mathrm{ml}$ and $1600 \mu \mathrm{g} / \mathrm{ml}$ when compared to standard drug diclofenac sodium. The compound 2-(2,3-dimethyl phenylamino)-N-(4-(4-hydroxyphenyl) thiazol-2-yl) benzamide (6g) also exhibited good anti-inflammatory activity at 400,800 and $1600 \mu \mathrm{g} / \mathrm{ml}$. Presence of methyl (6a), nitro (6c) and hydroxyl (6g) substituent in at 4-phenyl thiazole derivatives exhibited good anti-inflammatory activity when compared to other substituents. The percentage inhibition of protein denaturation compound $6 \mathrm{a}$ is $76.62 \%$ and $78.81 \%$ at 800 and $1600 \mu \mathrm{g} / \mathrm{ml}$ respectively and compound $6 \mathrm{c}$ is $71.20 \%$, $75.65 \%$ and $79.93 \%$ at 400,800 and $1600 \mu \mathrm{g} / \mathrm{ml}$ respectively showed better activity when compared to standard drug diclofenac sodium. All other compounds exhibited moderate anti-inflammatory activity compared with the standard. Results of the percentage of protein inhibition were shown in Table-2 and Fig.-3.

Tabel: 1-Physical Properties of Synthesized Compounds (6a-k)

\begin{tabular}{c|c|c|c|c|c}
\hline Compounds & $\mathrm{R}$ & $\begin{array}{c}\text { Molecular } \\
\text { Formula }\end{array}$ & $\begin{array}{c}\text { Molecular } \\
\text { Weight }\end{array}$ & $\begin{array}{c}\text { Melting } \\
\text { Point }\left({ }^{\circ} \mathrm{C}\right)\end{array}$ & $\begin{array}{c}\mathrm{R}_{\mathrm{f}} \\
\text { Value }\end{array}$ \\
\hline $6 \mathrm{a}$ & $4-\mathrm{CH}_{3}$ & $\mathrm{C}_{25} \mathrm{H}_{23} \mathrm{~N}_{3} \mathrm{OS}$ & 413.53 & $184-185$ & 0.69 \\
\hline $6 \mathrm{~b}$ & $4-\mathrm{OCH}_{3}$ & $\mathrm{C}_{25} \mathrm{H}_{23} \mathrm{~N}_{3} \mathrm{O}_{2} \mathrm{~S}$ & 429.53 & $186-188$ & 0.52 \\
\hline $6 \mathrm{c}$ & $4-\mathrm{NO}_{2}$ & $\mathrm{C}_{24} \mathrm{H}_{20} \mathrm{~N}_{4} \mathrm{O}_{3} \mathrm{~S}$ & 444.51 & $207-209$ & 0.48 \\
\hline $6 \mathrm{~d}$ & $\begin{array}{c}3-\mathrm{OCH}_{3} 4-\mathrm{OCH}_{3}, \\
5-\mathrm{CCH}_{3}\end{array}$ & $\mathrm{C}_{27} \mathrm{H}_{27} \mathrm{~N}_{3} \mathrm{O}_{4} \mathrm{~S}$ & 489.59 & $175-177$ & 0.71 \\
\hline $6 \mathrm{e}$ & $4-\mathrm{F}$ & $\mathrm{C}_{24} \mathrm{H}_{20} \mathrm{FN} \mathrm{F}_{3} \mathrm{OS}$ & 417.50 & $179-181$ & 0.63 \\
\hline $6 \mathrm{f}$ & $3-\mathrm{OCH}$ & $\mathrm{C}_{25} \mathrm{H}_{23} \mathrm{~N}_{3} \mathrm{O}_{2} \mathrm{~S}$ & 429.53 & $194-195$ & 0.53 \\
\hline $6 \mathrm{~g}$ & $4-\mathrm{OH}$ & $\mathrm{C}_{24} \mathrm{H}_{21} \mathrm{~N}_{3} \mathrm{O}_{2} \mathrm{~S}$ & 415.51 & $208-210$ & 0.42 \\
\hline $6 \mathrm{~h}$ & $4-\mathrm{Br}$ & $\mathrm{C}_{24} \mathrm{H}_{20} \mathrm{BrN}_{3} \mathrm{OS}$ & 478.40 & $200-202$ & 0.58 \\
\hline $6 \mathrm{i}$ & $4-\mathrm{Cl}$ & $\mathrm{C}_{24} \mathrm{H}_{20} \mathrm{ClN}_{3} \mathrm{OS}$ & 433.95 & $197-198$ & 0.68 \\
\hline $6 \mathrm{j}$ & $3-\mathrm{OCH}_{3}, 4-\mathrm{OH}$ & $\mathrm{C}_{25} \mathrm{H}_{23} \mathrm{~N}_{3} \mathrm{O}_{3} \mathrm{~S}$ & 445.53 & $218-220$ & 0.64 \\
\hline $6 \mathrm{k}$ & $3-\mathrm{OCH}_{3,4-\mathrm{OCH}}$ & $\mathrm{C}_{26} \mathrm{H}_{25} \mathrm{~N}_{3} \mathrm{O}_{3} \mathrm{~S}$ & 459.56 & $174-176$ & 0.45 \\
\hline
\end{tabular}

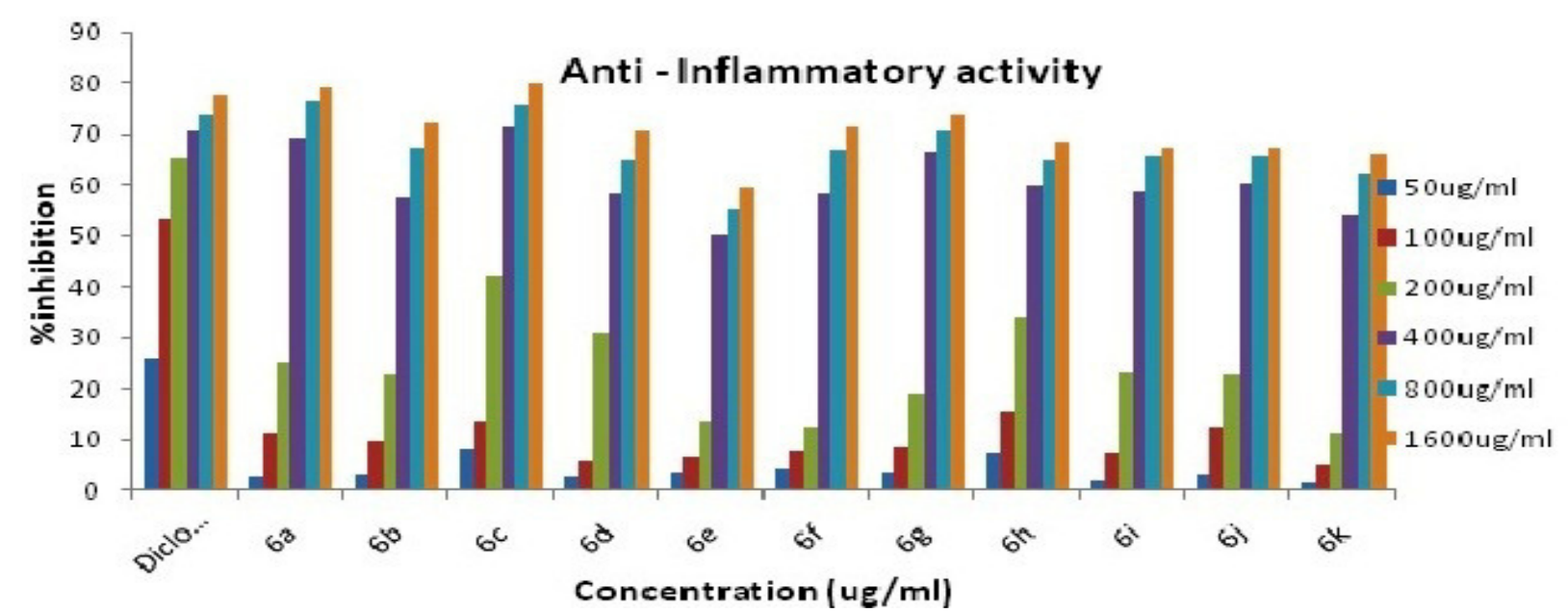

Fig.-3: Graphical Representation of Anti-inflammatory Activity of Substituted Thiazoles (6a-k)

Table-2: Anti-inflammatory Activity of Synthesized Compounds (6a-k)

\begin{tabular}{c|c|c|c|c|c|c|c}
\hline S. No. & Compound Code & \multicolumn{6}{|c}{ Inhibition of Denaturation (in \%) } \\
\hline & & 50 & 100 & 200 & 400 & 800 & 1600 \\
& & $\mu \mathrm{g} / \mathrm{ml}$ & $\mu \mathrm{g} / \mathrm{ml}$ & $\mu \mathrm{g} / \mathrm{ml}$ & $\mu \mathrm{g} / \mathrm{ml}$ & $\mu \mathrm{g} / \mathrm{ml}$ & $\mu \mathrm{g} / \mathrm{ml}$ \\
\hline 1. & $6 \mathrm{a}$ & 2.47 & 10.99 & 24.98 & 68.95 & 76.62 & 78.81 \\
\hline 2. & $6 \mathrm{~b}$ & 2.86 & 9.41 & 22.58 & 57.19 & 67.03 & 72.13 \\
\hline 3. & $6 \mathrm{c}$ & 7.60 & 13.18 & 41.58 & 71.20 & 75.65 & 79.93 \\
\hline 4. & $6 \mathrm{~d}$ & 2.29 & 5.74 & 30.63 & 57.92 & 64.66 & 70.50 \\
\hline 5. & $6 \mathrm{e}$ & 3.38 & 6.09 & 13.18 & 49.74 & 55.29 & 59.23 \\
\hline 6. & $6 \mathrm{f}$ & 3.96 & 7.41 & 12.41 & 57.98 & 66.53 & 71.02 \\
\hline
\end{tabular}


RASĀYAN J. Chem.

Vol. 12 | No. 1 |366 - 371| January - March | 2019

\begin{tabular}{c|c|c|c|c|c|c|c}
\hline 7. & $6 \mathrm{~g}$ & 3.38 & 8.17 & 18.91 & 66.10 & 70.49 & 73.56 \\
\hline 8. & $6 \mathrm{~h}$ & 7.06 & 15.05 & 33.79 & 59.55 & 64.64 & 68.49 \\
\hline 9. & $6 \mathrm{i}$ & 1.73 & 6.78 & 22.99 & 58.51 & 65.51 & 66.92 \\
\hline 10. & $6 \mathrm{j}$ & 2.68 & 12.26 & 22.35 & 60.10 & 65.46 & 66.93 \\
\hline 11. & $6 \mathrm{k}$ & 1.17 & 4.86 & 11.20 & 54.10 & 62.10 & 65.70 \\
\hline 12. & Diclofenac sodium & 25.65 & 53.21 & 65.04 & 70.28 & 73.50 & 77.27 \\
\hline
\end{tabular}

\section{CONCLUSION}

A series of 2,4-disubstituted thiazole derivatives (6a-k) were synthesized by a condensation reaction between 2-amino 4-substituted phenyl thiazole and 2-(2,3-dimethylanilino)benzoic acid by using DCC/DMAP coupling. These synthesized thiazole derivatives $6(\mathrm{a}-\mathrm{k})$ were evaluated for their in vitro antiinflammatory activity using albumin denaturation method using diclofenac sodium as standard drug. All the synthesized compounds showed significant anti-inflammatory activity. Among these compounds 2(2,3-dimethylphenylamino)-N-(4-(4-methyl phenyl) thiazol-2-yl)benzamide (6a) and 2-(2,3dimethylphenylamino)-N-(4-(4-nitrophenyl) thiazol-2-yl)benzamide (6c) showed better antiinflammatory activity when compared to standard diclofenac sodium at both $800 \mu \mathrm{g} / \mathrm{ml}$ and $1600 \mu \mathrm{g} / \mathrm{ml}$ when compared to the standard drug diclofenac sodium. The compound 2-(2,3-dimethylphenylamino)-N(4-(4-hydroxyphenyl) thiazol-2-yl) benzamide (6g) also exhibited good anti-inflammatory activity at 400, 800 and $1600 \mu \mathrm{g} / \mathrm{ml}$. All other derivatives exhibited mild to moderate anti-inflammatory activity compared with the standard.

\section{ACKNOWLEDGMENT}

The authors are thankful to the "Vagdevi College of Pharmacy and Research center, Nellore-524 346, Andhra Pradesh, A.P, India, for providing required facilities.

\section{REFERENCES}

1. J. Quiroga, P. Hernandez, B. Insuasty, R. Abonia, J. Cobo, A. Sanchez, M. Nogueras and J.N. Low, J. Chem. Soc., Perkin Trans., 1(4),555 (2002), DOI: 10.1039/b109676a.

2. I. Hutchinson, S. A. Jennings, B. R. Vishnuvajjala, A. D. Westwell and M. F. G. Stevens, J. Med. Chem., 45, 744(2002), DOI: 10.1021/jm011025r.

3. M. T. Chhabria, S. Patel, P. Modi and P. S. Brahmkshatriya, Curr. Top. Med. Chem., 16, 2841 (2016), DOI: $10.2174 / 1568026616666160506130731$.

4. K. Sampath, K. Karthik, R. Sivahari, P. Arunkumar, Rasayan J. Chem., 10, 1184 (2017), DOI: 10.31788/RJC.2018.1143080.

5. P. Karegoudar, M. S. Karthikeyan, D. J. Prasad, M. Mahalinga, B. S. Holla and N. S. Kumari, Eur. J. Med. Chem, 43, 261(2008), DOI: 10.1016/j.ejmech.2007.03.014.

6. P. Vicini, A. Geronkiaki, M. Incerti, F. Zani, J. Dearden and M. Hewitt, Bioorg. Med. Chem., 16, 3714 (2008), DOI: 10.1016/j.bmc.2008.02.001.

7. R. P. Karuvalam, K. R. Haridas, S. K. Nayak, T.N. Guru Row, P.Rajesh, R.Rishikesan, N. Suchetha Kumari, Eur. J. Med. Chem., 49,172 (2012), DOI: 10.1016/j.ejmech.2012.01.008.

8. A Andreani, M. Rambaldi, A. Leoni, A. Locatelli, R. Bossa, M. Chiericozzi, I. Galatulas and G. Salvatore, Eur. J. Med. Chem, 31, 383 (1996), DOI: 10.1016/S0223-5234(99)80015-4.

9. B Jiang and X.- H. Gu, Bioorg. Med. Chem., 8, 363 (2000), DOI: 10.1016/S0968-0896(99)00290-4.

10. Pradeep Kate, Shashikant Gaikwad, Tukaram Lokhande, Abdul Shaikh, Babasaheb Sonawane, Praffula Choudhari and Madhusudan Bachute, Rasayan J. Chem., 11(4), 1441(2018), DOI: $10.31788 /$ RJC.2018.1143080.

11. Yasser Hussein Eissa Mohammed, Vikas H. Malojirao, Prabhu Thirusangu, Mohammed AlGhorbani, B.T. Prabhakar and Shaukath Ara Khanum, Eur. J. Med. Chem., 143, 1826 (2018), DOI: 10.1016/j.ejmech.2017.10.082.

12. Jignasa K. Savjani, Suja Mulamkattil, Bhavesh Varia and Snehal Patel, European Journal of Pharmacology, 801, 28 (2017), DOI: 10.1016/j.ejphar.2017.02.051.

13. M. Gnana, Ruba Priya, K. Girija and N. Ravichandran, Rasayan J. Chem., 4, 418 (2011).

[RJC-5069/2018] 